\title{
Politique
}

\section{Crise sociale, mouvements sociaux et pratiques du changement} social

\section{Louis Maheu}

Numéro 4, automne 1983

Crise et changements idéologiques

URI : https://id.erudit.org/iderudit/041001ar

DOI : https://doi.org/10.7202/041001ar

Aller au sommaire du numéro

Éditeur(s)

Société québécoise de science politique

ISSN

0711-608X (imprimé)

1918-6584 (numérique)

Découvrir la revue

Citer cet article

Maheu, L. (1983). Crise sociale, mouvements sociaux et pratiques du changement social. Politique, (4), 27-54. https://doi.org/10.7202/041001ar d'utilisation que vous pouvez consulter en ligne.

https://apropos.erudit.org/fr/usagers/politique-dutilisation/ 


\title{
Crise sociale, mouvements sociaux et pratiques du changement social
}

\author{
Louis MAHEU \\ Université de Montréal
}

Il y a maintenant un thème sur lequel il n'est nul besoin d'insister, si l'on veut qu'il pénètre les consciences et les imaginations contemporaines: soit celui de la crise. Tout au plus, rencontre-t-on encore de temps à autre un dernier carré de fideles qui souhaiteraient convaincre que la crise ne serait qu'économique. Avec la reprise des affaires, avec un meilleur contrôle des problèmes structurels de l'accumulation intensive du capital, si tant est d'ailleurs que cela était davantage possible que souhaitable, les soi-disant autres dimensions de la crise seraient vite balayées. Et la place vide, ainsi, à l'évidence serait quand même pleine de la preuve que tout dans cette crise n'est qu' «affaire», expression appropriée s'il en est une, d'économie et de capital.

Mais les déloyaux envers semblable interprétation sont plus nombreux maintenant à penser cette crise comme un phénomène total dont les racines plongent dans le culturel et le politique. Certains vont même jusqu’à parler de crise d'hégémonie sociale. C'est de ce côté, bien sûr, qu'il faut jeter son regard. Encore que, ce point de vue adopté, l'introspection gagnerait à être plus perspicace. Et qu'est-ce qu'il y a d'autre alors à scruter? Une crise, surtout totale, on le sait depuis un bon moment, est intéressante aussi en ce qu'elle comporte, déclenche et en- 
gage des perspectives de redressements qui risquent de se muer, avec un bon nombre d'ajustements il est vrai, en éléments structurels fondamentaux d'un nouveau système de rapports qui est à se mettre en place.

Dans les pages qui suivent, on fera allusion aux pratiques sociales de groupes de base, de mouvements sociaux partiels, pratiques sociales dites souvent alternatives, notamment par rapport aux pratiques sociales plus institutionalisées du mouvement ouvrier et syndical. On retracera certaines incitations aux développements de ces pratiques au sein des facteurs structurels de la crise, crise politique, économique et culturelle. Et simultanément, ces incitations structurelles se révélant des enjeux conflictuels en train de se démarquer plus nettement au sien des sociétés industrielles avancées, on verra si elles participent de la restructuration de rapports sociaux dont le champ englobe des revendications, des contestations, des luttes rompant quelque peu avec les pratiques sociales antérieures. Revendications, contestations, luttes sociales qui seraient vraisemblablement liées à des voies de changement social en instance d'émergence et de développement.

On examinera d'abord les contraintes structurelles des sociétés industrielles avancées véhiculant des enjeux conflictuels qui mettent en scène des groupes de base, des mouvements sociaux partiels. On précisera aussi comment ces enjeux conflictuels ouvrent certaines voies au changement social et quelles sont les orientations qu'il peut emprunter. On mesurera alors en quoi ces pratiques émergentes de changement social, dont la conceptualisation théorique demeure à parfaire, se distinguent de pratiques plus connues de rapports de classe et de changement social. Enfin, le cheminement parcouru invitera à discuter quelques aspects des faits de conscience liés aux pratiques sociales des groupes de base et certains traits de leur spécificité politi- 
que plus immédiate eu égard au fonctionnement d'un système politique.

Le revenu du citoyen et l'appropriation étatique du tissu social

Nous n'avons pas l'intention de procéder à un relevé, systématique et des plus compréhensifs, de l'ensemble des contraintes structurelles, des contradictions qui enserrent et conditionnent les pratiques sociales. Un tel exercice est d'ailleurs futile. Rendre compte de toutes les contraintes structurelles à l'œuvre au sein d'un système social dépasse les capacités de tout schéma d'analyse. Il est plutôt question d'identifier certains enjeux structurels plus particuliers permettant de canaliser le regard vers les pratiques sociales d'agents n'appartenant pas à la classe ouvrière, au sens classique du terme, mais constituant ce que certains nomment soit des mouvements sociaux, soit des groupes de base, soit encore des groupes alternatifs. Les éléments structurels qui seront donc retenus ici permettront d'aller plus directement à la caractérisation des pratiques émergentes, souvent dénommées alternatives, du changement social.

Beaucoup d'observateurs des sociétés industrielles avancées se sont attardés à la notion de fordisme, d'abord vraisemblablement utilisée par A. Gramsci, pour rendre compte de l'évolution récente de ces sociétés. Ils visaient alors à les qualifier du point de vue de l'accumulation intensive du capital qui était le plus souvent liée à la déqualification du travail et à une augmentation du travail mort causée par l'intervention de la science et de la technologie dans le procès et de production et de circulation des marchandises ${ }^{1}$. Que pareil développement des forces

1. Voir à ce sujet, A. D. Magaline, Lutte de classe et dévalorisation du capital. Paris, Maspéro, 1975; H. Braverman, Labor and Monopoly Capital; the Degradation of Work in the 20th Century, New York, Monthly Review, 1974; B. Coriat, Science, Techni- 
productives sous-tendant l'accumulation du capital n'ait pu être assuré par le seul capital privé est maintenant un trait bien documenté des sociétés industrielles avancées. L'État, le capital public, a été largement mis à contribution. De bien des manières, il a conforté les opérations préparant directement ou indirectement l'accumulation intensive du capital. Les subventions directes aux entreprises en difficulté financière ou en voie de réaliser des expansions d'opération dans des régions économiques en butte à une faible croissance socio-économique ne sont pas seules en cause. L'État intervient et est intervenu dans nombre d'opérations de financement des infrastructures nécessaires au procès de développement des forces productives : le système scolaire, le système de la recherche-développement, le système de santé. On sait pertinemment qu'il fut très directement concerné par les processus mêmes de dévalorisation de fractions du capital, fractions engagées dans le financement de besoins ou de programmes sociaux de tous ordres ne rendant pas, le plus souvent, de profits égaux aux sommes investies ${ }^{2}$. De plus, dans le processus de circulation du capital, les agents touchant le taux de rendement maximal de semblables investissements, ne relevaient pas en général des appareils économiques de la société politique.

Mais le plus important ici est bien d'observer que l'intervention de l'État n'était fonction ni du seul procès de production, de circulation du capital, ni des seules pressions sur l'État

que et capital, Paris, Seuil, 1976 et l'Atelier et le chronomètre. Essai sur le taylorisme, le fordisme et la production de masse, Paris, Bourgeois, 1979; A. Lipietz, Crise et inflation: Pourquoi? Paris, Maspéro, 1979, «Marchandise, autogestion et "Capitalisme organisé»", Les Temps Modernes, no. 391, février 1979 et «La double complexité de la crise», Les Temps modernes, no. 407, juin 1980.

2. Sur ce thème, voir, A. D. Magaline, op. cit; P. Boccara, Sur la mise en mouvement du capital, Paris, Éditions sociales, 1978; Collectif, Traité marxiste d'économie, le capitalisme monopoliste d'État, 2 tomes, Paris, Éditions sociales, 1971: D. Harris, "On Marx's schema of reproduction and accumulation", Journal of Political Economy, Vol. 80, 1972. 
et les appareils d'État des classes économiquement dirigeantes. En effet, dans beaucoup de sociétés industrielles avancées, l'intervention de l'État se soldait par sa contribution aux revenus, directs ou indirects, d'un nombre croissant et important d'agents sociaux $^{3}$. S. Bowles et $\mathrm{H}$. Gintis ont très longuement et systématiquement documenté semblables facettes du développement socio-économique contemporain des États-Unis ${ }^{4}$. C'est ainsi qu'ils relèvent aux États-Unis, dans l'après-deuxième guerre mondiale, une relation entre le processus d'accumulation du capital et le fonctionnement de la démocratie libérale impliquant une "association" nouvelle entre le capital et le travail. Cette restructuration des relations entre ces deux classes sociales est soustendue par un ensemble de législations sociales et manifeste combien l'État et son fonctionnement sont devenus un terrain important de la lutte des classes. Cette dernière, dans ce contexte, passe notamment par les enjeux des pratiques et des politiques de distribution des ressources: luttes concernant les salaires réels, le niveau des taxes, la fixation des loyers, la nature et le taux des dépenses publiques d'aide sociale.

Une part non négligeable des principaux avantages distributionnels obtenus par les travailleurs ne résultait pas d'une confrontation directe avec le capital, mais venait plutôt de l'État. C'est que l'intervention de celui-ci contribuait à une socialisation de la consommation au sens où certains services collectifs tendaient à être retirés de la sphère de circulation des marchandises, l'éducation par exemple, et au sens où l'intervention de

3. A. Lipietz, Crise et inflation..., op. cit... Voir encore à ce sujet, M. Decaillot, E. Preteceille, J. Terrail, Besoins et modes de production, Paris, Éditions sociales, 1977; P. Gruet, Besoins populaires et financement public, Paris, Éditions sociales, 1976.

4. Les propos qui suivent font directement référence au texte suivant de $\mathrm{S}$. Bowles et H. Gintis, «La crise du capitalisme démocratique libéral aux États-Unis», Les Temps Modernes, nos. 430 et 431, mai et juin 1982; voir aussi S. Bowles et al. Beyond the Wasteland: a Democratic Alternative to Economic Decline, New-York, Double Day Anchor Press, 1983. 
l'État faisait suite à de fortes revendications en faveur d'un contrôle du prix de la consommation de certains équipements, les loyers et les transports par exemple, ou en faveur de la protection de ressources collectives, des espaces verts, de l'environnement. Mais les avantages distributionnels pouvaient aussi venir de l'intervention de l'État visant le maintien d'un niveau de vie minimal pour les personnes socio-économiquement démunies relevant directement de programmes d'aide et d'assistance sociale. Finalement, toutes ces dimensions d'une socialisation de la consommation participent de l'évolution tout à fait déterminante des revenus du citoyen.

À côté donc des revenus tirés d'un salaire, ne peuvent être ignorées les ressources, non négligeables et en croissance procentuelle soutenue, constitutives du revenu ou du salaire du citoyen et fournies par les programmes d'aide financière mis au point par l'État.

«En $1973,44 \%$ du nombre total de foyers ont reçu une aide financière du gouvernement atteignant en moyenne $\$ 3368.00$ et représentant $24 \%$ de leur revenu global moyen; les personnes âgées ont reçu quant à elles une aide financière représentant 39\% de leur revenu global moyen ${ }^{5}$.»

Et S. Bowles et H. Gintis de soutenir encore que le rôle joué par le conflit distributionnel dans le processus récent d'accumulation du capital fut déterminant et qu'en général les capitalistes en ont quand même fait les frais dans une proportion significative. Le conflit distributionnel et le revenu du citoyen ont notamment ralenti le processus de croissance capitaliste et ont contribué à rendre moins efficaces, dans le cycle de reproduction du capital, les phénomènes de crise et les pressions exercées par l'armée de réserve sur les travailleurs, les chômeurs et les grévistes. Et il en fut ainsi parce que les classes sociales défavorisées ont mené de

5. S. Bowles, H. Gintis, «La crise du capitalisme...», op. cit., 2145. 
nombreuses luttes reliées à l'axe distributionnel de la lutte des classes. Du coup, le conflit distributionnel et les pratiques qui en ont découlé ont eu un impact sur l'équilibre des rapports entre le capital et le travail et ils ont constitué un rouage important de leur reproduction.

Cette démonstration s'appliquant d'abord aux États-Unis peut-elle rendre compte de la situation canadienne et québécoise? On peut émettre l'hypothèse que cette tendance se prolonge au sein de nos formations sociales, étant donné les nombreuses similitudes caractérisant ces diverses sociétés industrielles avancées. Mais un autre trait de ce processus mérite d'être mis tout particulièrement en relief. Il implique tout d'abord que l'on distingue dans l'axe distributionnel de la lutte des classes, selon l'expression de S. Bowles et H. Gintis, les pressions dirigées vers les salaires réels, des revendications relatives au revenu du citoyen. Et alors, on notera que l'expansion du revenu du citoyen - suite aux pressions exercées sur la société politique et l'État de la démocratie libérale en faveur de la conquête des droits sociaux des citoyens - de même que l'intervention étatique au niveau des biens de consommation collective ont contribué à tisser des rapports tels entre certaines couches de population et l'État que celui-ci est devenu le pourvoyeur assez direct des conditions socio-économiques de ces couches. Les pressions exercées sur les appareils de la société politique et l'État pour qu'ils augmentent les ressources et modifient l'application de leurs multiples programmes sociaux ont été d'abord et avant tout le fait, non pas uniquement de la classe ouvrière au sens classique du terme et du mouvement syndical, mais bien de groupes de base, le cas échéant de mouvements sociaux, défendant les groupes qui dépendent plus largement que d'autres du revenu du citoyen ou encore se faisant les porte-parole de certains groupes d'usagers des biens de consommation collective. 
Ces groupes mènent contre les appareils d'État, quasipropriétaires et quasi-gestionnaires des ressources et des biens conditionnant leur niveau socio-économique de vie, contre les appareils d'État fixant finalement le niveau du revenu du citoyen, des luttes qui pourraient être qualifiées, par analogie, de syndicales. Ces dernières possèdent à la fois leur volet défensif de luttes pour le maintien, sinon l'expansion du revenu et leur volet offensif d'élargissement d'un contre-pouvoir contestant l'aliénation et la perte d'autonomie imposées par les appareils de la société politique et l'État. Force est d'admettre qu'il s'agit là d'un terrain tout à fait stratégique de mobilisation de groupes de base, de mouvements sociaux partiels constitutifs de classes dominées élargies, du peuple en situation de rapports sociaux de domination ${ }^{6}$.

L'interventionnisme économique de l'État de la démocratie libérale, n'est aucunement fonction, dans le processus social global de l'accumulation intensive du capital, des seules prescriptions des procès de production de biens et de marchandises et de circulation du capital ou encore des seules pressions des classes économiquement dirigeantes. Bien au contraire, l'interventionnisme étatique résulte des conquêtes sociales des classes dominées relatives notamment à la distribution des ressources. Mais quelles que soient les forces sociales placées en amont de l'interventionnisme étatique dans le champ économique au sens large du terme, il faut noter, que cette intervention, est aussi politique. Elle est tout d'abord politique parce qu'elle a été préparée sur le terrain politique: elle est le produit d'un État, d'un

6. Cette composante, appelée lutte syndicale, de conduites collectives de groupes de base a notamment été mise en relief dans une étude portant sur des coopératives québécoises non lucratives en habitation; voir S. Bernard, M. Fafard, L. Maheu, C. Saucier, Coopératives en babitation et intellectuels des groupes de ressources techniques; le rapport à l'espace: un probleme de solidarité et de changement social, Montréal, Département de sociologie, Université de Montréal, 1980. Une version révisée et corrigée de ce texte sera publiée prochainement. 
système politique de la démocratie libérale où ont pu être affirmés et conquis certains droits des citoyens. Mais l'intervention de l'État est aussi politique parce qu'elle met en branle les appareils de la société politique, et par là, la bureaucratie d'État.

Du coup, plusieurs autres traits de l'interventionnisme étatique, peuvent être dégagés. Cet interventionnisme produit de l'intégration sociale: il démarginalise d'importantes couches sociales d'agents sociaux, de citoyens, instaurant entre ceux-ci et l'État un intense tissu d'interrelations sociales. Ces interrelations sociales assurent alors des fonctions telles qu'elles entraînent une meilleure connaissance des sujets, une information détaillée sur leurs conditions de vie, une surveillance plus étroite de leur cheminement socio-économique. Cet interventionnisme étatique suppose aussi des politiques et des pratiques autoritaires de gestion du social. Les grandes décisions sont entre les mains de la technocratie d'État, et leur exécution, sous forme de politiques et de programmes sociaux est confiée aux appareils de la bureaucratie d'État. Ces appareils imposent le plus souvent à la clientèle sociale qui les fréquente le recours à une expertise professionnelle en raison de la quantité et de la complexité des informations à maîtriser pour avoir accès à ces programmes so$\operatorname{ciaux}^{7}$.

On pourrait ajouter encore à ces traits politiques d'interventionnisme étatique: mais leur convergence d'ensemble vers des politiques autoritaires de gestion du social n'a plus besoin d'être davantage détaillée. Soit dans leurs rapports directs avec les appareils d'État, soit dans leurs rapports indirects, des fonc-

7. Sur l'ensemble de ces thèmes, voir $\mathrm{C}$. Lefort, L'invention democratique: les limites de la domination totalitaire, Paris, Fayard 1981 et Éléments d'une critique de la bureaucratie, Paris, Gallimard, 1979; M. Weber, Économie et Société, Paris, Plon, 1971; P. Clastres, La société contre l'État, Paris, Minuit, 1975; et sur la question des rapports entre les appareils d'État et leur clientèle sociale au moyen des expertises professionnelles, voir S. Bernard, M. Fafard, L. Maheu, C. Saucier, Coopératives en babitation..., op. cit. 
tions et des agents intellectuels s'interposent entre la clientèle sociale et les appareils; avec eux, diverses couches sociales sont soumises à une participation dépendante face à des politiques autoritaires de gestion du social ${ }^{8}$. On pourrait alors soutenir, et c'est là une idée à laquelle des développements ont été consacrés ailleurs ${ }^{9}$, qu'une contradiction majeure des sociétés industrielles avancées, qu'un de leurs enjeux conflictuels clés concerne précisément l'opposition entre, d'une part, une appropriation étatique du tissu social et, d'autre part, une appropriation collective de ce même tissu social.

Il s'agit alors de souligner qu'à l'interventionnisme étatique est jouxtée une appropriation étatique du tissu social reposant sur les politiques autoritaires et bureaucratiques de gestion du social. Et face à cette appropriation étatique du tissu social, des luttes de groupes de base, de mouvements sociaux partiels se dessinent de plus en plus nettement: elles désignent et balisent tout à la fois l'enjeu d'une appropriation collective du tissu social. Des recherches antérieures ont permis d'identifier deux traits majeurs de ces luttes: d'une part elles visent une plus grande autonomie, une volonté d'auto-contrôle à la base, d'aires du tissu social - par exemple, certains aspects des soins de santé, diverses facettes de la consommation d'équipements collectifs comme le transport en commun ou les espaces verts, ou certaines formes de consommation d'habitat comme les coopératives à but non lucratif - et d'autre part, des formes de solidarité et de sociabilité relativement nouvelles, plus ludiques et communautaires, entre membres de groupes de base. Ces formes de solidarité et de sociabilité contribuent directement à la

8. Voir à ce sujet, A. Touraine, La voix et le regard, Paris, Seuil, 1978, Production de la société. Paris, Seuil, 1973 et La Société post-industrielle, Paris, Denoël, 1969.

9. Ce thème est traité plus longuement dans un autre texte qui paraîtra prochainement, L. Maheu, «Les mouvements de base et la lutte contre l'appropriation étatique du tissu social », Sociologie et sociétés. Vol. 15 no. 1, 1983. 
conquête d'une identité socio-culturelle du groupe d'agents sociaux en lutte ${ }^{10}$.

Dans la mesure même où il serait fondé de voir dans l'opposition entre l'appropriation étatique et l'appropriation collective du tissu social un enjeu conflictuel majeur des sociétés industrielles avancées, le positionnement social des groupes de base, des mouvements sociaux en termes de politique et de pratique du changement social, serait déterminant.

\section{L'articulation problématique entre le culturel} et le politique: problèmes d'identité socio-culturelle

Il est temps de délaisser, pour un moment, les problèmes que nous avons situés à la jonction de l'interventionnisme économique étatique et du fonctionnement du système politique à régime démocratique libéral. Ce sera pour plonger plus directement dans l'univers même de la culture et retracer, par ce cheminement, des problèmes d'articulation et de désarticulation du culturel et du politique qui devraient nous ramener à certains aspects des thèmes discutés précédemment. On doit à un travail provocant de Daniel Bell - «The cultural contradictions of Capitalism ${ }^{11}$ - un certain nombre de réflexions percutantes à propos du devenir du capitalisme contemporain. Travail provocant parce qu'il stimule l'imagination par des interrogations riches et fascinantes et à la fois parce qu'il nourrit l'impatience et la critique par une trop fréquente naïveté couplée à un manque déplorable de rigueur. On le sait, et Daniel Bell le rappellera fort à propos, que le mode de production capitaliste est forte-

10. Sur la notion d'appropriation collective du tissu social, voir L. Maheu, «Les mouvements de base...», Op. cit. et S. Bernard, M. Fafard, L. Maheu, C. Saucier, Coopératives en babitation.... op. cit...

11. D. Bell, The cultural Contradictions of Capitalism. New York, Basic Books, 1978, Les développements qui suivent font directement référence au texte de D. Bell. 
ment sous-tendu par une recherche effrénée de l'individualisme, notamment dans le champ économique. Individualisme des entrepreneurs, des producteurs capitalistes qui, comme Max Weber l'avait précisé, fonderont leurs pratiques sur une éthique de la responsabilité et un ascétisme puritain du devoir à accomplir, le succès matériel obtenu signalant combien Dieu, dans leurs ouvres mêmes, savait reconnaître et faire reconnaître les siens. Et D. Bell d'ajouter que cette culture bourgeoise de la responsabilité individuelle, de l'ascétisme, culture de la rationnalité et du pragmatisme, fut très rapidement prise d'assaut par une culture moderniste, elle aussi cultivant en quelque sorte l'individualisme. Apparaît donc un individualisme du moi absolu, opposant à tout devoir ascétique et à toute rationalité, l'affirmation, la conquête du moi absolu basée sur le droit à l'expérience, la négation de la contrainte, de la convention et de la tradition qui rendraient impossible le cheminement vers l'authenticité du moi. Un certain hédonisme vint rapidement contrecarrer aussi l'éthique de la responsabilité et l'ascétisme puritain. Du mouvement surréaliste des années '20 et '30 à la contre-culture la plus récente se manifeste cette culture moderniste du moi absolu.

Et ces tendances culturelles devaient être encore plus exacerbées quand la logique des catégories marchandes et capitalistes, la logique de la production, circulation et consommation du capital, les rejoignait et contribuait systématiquement à les matérialiser. D'une part, les signes de la réussite au sein des classes possédantes n'étaient plus la seule accumulation ascétique du capital pour l'investissement, mais la conquête de statuts sociaux et la maîtrise de niveaux de plus en plus élevés et luxuriants de consommation de biens et de services. Et d'autre part, la culture moderniste fondée sur la recherche de l'inédit, du non-conventionnel comme styles de vie et modèles culturels, pénétrait davantage le tissu social et parvenait à marquer plu- 
sieurs facettes de la culture de masse au moyen du caractère marchand du nouveau, de l'anti-conventionnel, du nontraditionnel. Le style culturel du «radical chic», selon l'expression de Bell ${ }^{12}$, rejoint une bonne fraction de la masse cultivée pratiquant des modes de vie supposément libres tout en profitant d'emplois bien rémunérés dans un système économique poussant à la conquête de statuts sociaux et à la surconsommation. La logique des catégories marchandes bientôt gagne la culture moderniste, y compris sa région contreculturelle, et en produit une appropriation capitaliste de même qu'elle en renverse le sens et la direction. Et l'ensemble des mass-media, de l'industrie des communications, n'ont pas été pour peu dans cette exacerbation du moi, des pulsions, de la recherche manipulée du plaisir et dans la prolifération des ressources consacrées à cultiver, sous de multiples angles, l'individualité du citoyen.

Il est toutefois étonnant de ne pas trouver chez Bell de mise en relation de cet abus marchand de l'individualité avec les politiques autoritaires de gestion non seulement du social, mais encore des personnalités, des plaisirs, des pulsions générées par les formations sociales capitalistes et les systèmes politiques à démocratie libérale. Il est vrai qu'en ce domaine les formations sociales dites socialistes ont connu, elles aussi, une évolution des plus surprenante. Mais pourtant au sein des formations sociales capitalistes, non seulement la recherche du profit et du rendement a-t-elle systématiquement compromise, au travers même des abus marchands de l'individualité, la vraie libération du moi et des pulsions, mais encore cette tendance fut consolidée par le fonctionnement des agences de socialisation et d'intégration sociale qui ont produit et reproduit l'uniformité sociale, l'intégration socio-institutionnelle au moyen des nombreuses mailles du

12. D. Bell, ibid, 20. 
filet des appareils de la société politique. On est loin alors de ce que $\mathrm{R}$. Bahro assigne comme objectif premier à une révolution culturelle, soit l'actualisation «d'un itinéraire vers le dedans» en mesure de rompre avec les contraintes sociales, politiques, mais aussi avec les catégories marchandes des sociétés capitalistes et non capitalistes, de faire intervenir «un surplus de conscience " comme force révolutionnaire ${ }^{13}$.

De nombreuses études sur le pouvoir et sur les tendances uniformisantes, unidimensionnelles de la croissance des personnalités au sein des sociétés capitalistes contemporaines ont bien illustré comment l'État et l'ensemble de la société politique n'étaient point étrangers à la manipulation des désirs, des pulsions, des personnalités et de leurs besoins de croissance. Ces instances, à leur manière, confortent les pressions déjà engagées par les lois de la compétition et l'impératif du rendement relevant des catégories marchandes capitalistes, les unes et les autres convergeant vers ce que certains appellent le dressage des corps et des paroles et le quadrillage de l'existence ${ }^{14}$.

On est donc en mesure ici de dégager une contrainte structurelle majeure, une contradiction des sociétés industrielles avancées qui sont simultanément en proie à une recherche et à une promotion de l'individualité mais aussi, en même temps, à

13. Voir à ce sujet, R. Bahro, L'Alternative, Pour une critique du socialisme existant réellement, Paris, Stock 2, 1979 surtout la troisième partie, et le très intéressant commentaire que H. Marcuse consacrait à ce bouquin: "Protosocialisme et capitalisme avancé; Essai sur la méthodologie de la révolution à la lumière des prémisses de $R$. Bahro», Les Temps Modernes, no. 394, mai 1979.

14. Sur l'ensemble de ces thèmes, voir H. Marcuse, L'Homme unidimensionnel, Paris, Minuit, 1968; M. Foucault, Surveiller et punir. Naissance de la prison, Paris, Gallimard, 1975 et Histoire de la sexualité 1: La volonté de savoir, Paris, Gallimard, 1976 et Les mots et les choses, Paris, Gallimard, 1966; G. Deleuze et F. Guattari, Capitalisme et schizophrénie 1: L'anti-oedipe, II Mille Plateaux, Paris, Minuit, 1972 et 1980; J.-F. Lyotard, La condition post-moderne, Paris, Minuit, 1979; M. Gauchet, G. Swain, La pratique de l'esprit bumain. L'institution asilaire et la révolution démocratique, Paris, Gallimard, 1980; F. Castel, R. Castel, A. Lovell, La société psychiatrique avancée; le modele américain, Paris, Grasset, 1979. 
un abus marchand de cette individualité et à une augmentation du contrôle social au moyen d'un épaississement notable des instances et des appareils de l'intégration sociale et de l'uniformisation des personnalités. Il s'agit donc là d'une articulation problématique du culturel et du politique qui désigne des enjeux conflictuels, voire des agents sociaux - surtout ceux dont l'être social ou l'identité socio-culturelle demeurent un enjeu ouvert et encore arbitraire, et par là conflictuel - pouvant être déterminants pour les pratiques de changement social au sein des sociétés industrielles avancées.

Cette articulation du culturel et du politique, articulation tout à fait problématique dans la mesure où elle est le siège d'une contradiction majeure des sociétés industrielles avancées, doit être maintenant ramenée à une autre forme d'articulation du culturel et du politique. Qu'il nous soit permis, pour un court moment, de revenir aux luttes sociales que nous avons liées à l'axe distributionnel de la lutte des classes pour souligner qu'elles empruntaient les voies de pression exercées sur les salaires réels, sur les taxes, sur les programmes gouvernementaux et les revenus du citoyen. Nous n'avons peut-être pas assez précisé combien ces luttes, et surtout celles concernant le revenu du citoyen, étaient fonction de traits particuliers d'un régime politique appartenant à la démocratie libérale. Or, et c'est là un trait soulevé autant par $\mathrm{S}$. Bowles et $\mathrm{H}$. Gintis que par D. Bell, les systèmes politiques de la démocratie libérale sont particulièrement sensibles aux luttes menées au nom des droits du citoyen.

Mais on est forcé d'admettre qu'imperceptiblement, au point de départ, puis de plus en plus clairement et systématiquement par la suite, les pressions exercées en faveur des droits des citoyens l'ont été en fonction de groupes d'agents sociaux menaçant chaque fois les fonctions de légitimation de l'État. Et dès lors faut-il admettre cette contradiction des systèmes politi- 
ques de la démocratie libérale officiellement tournés vers le traitement des droits et des problèmes politiques des citoyens mais confrontés finalement aux pressions politiques de groupes d'agents sociaux qui leur arrachent et leur imposent l'exercice de leurs droits collectifs.

On doit aussi préciser que les groupes sociaux qui ont surpris le fonctionnement du système politique des démocraties libérales n'étaient point, en général, des groupes déjà marqués socialement. Les revendications et les pressions sociales de certains groupes incluant, par exemple, le mouvement ouvrier, se sont, elles, graduellement institutionnalisées à travers divers mécanismes d'arbitrage confirmant dans leur vocation des législations et des appareils de la société politique devant garantir et surveiller la paix sociale. Par contre, les fonctions de légitimation de l'ordre social exercées par l'État se sont révélées plus vulnérables quand les pressions sont venues de groupes sociaux socialement plus indéfinis et jusqu'alors mal identifiés. Il est ici principalement question des couches de population dont le positionnement social est plus directement fonction du revenu, du salaire du citoyen ou encore de ces couches sociales plus directement mobilisées par des enjeux conflictuels à titre d'usagers de biens et de services de consommation collective. Les revendications et les pressions venant de ces groupes sociaux ne se définissent pas prioritairement par leur appartenance à des systèmes de rapports sociaux de production et de circulation de biens et de marchandises, rapports sociaux liant et opposant des agents aux intérêts antagonistes. Le fonctionnement du système politique des démocraties libérales fut aussi marqué par des revendications relatives aux droits de groupes sociaux cherchant à conquérir leur être social, leur identité socio-culturelle. C'est le cas, par exemple, de groupes de jeunes, de femmes, de personnes âgées, de groupes ethniques, de groupes définis par la couleur de leur 
peau, ou encore de groupes se distinguant par leurs orientations sexuelles.

Et ici précisément, on peut parler à nouveau d'une articulation du culturel au politique. Tous ces groupes d'agents sociaux - groupes constituant la base sociale de mouvements sociaux partiels - sont mus par une volonté de conquête d'une identité socio-culturelle de groupes en lutte. Le sens même de leurs luttes et de leurs pratiques conflictuelles ne peut être dissocié de cette recherche visant à rendre légitime et à imposer l'enjeu même des conflits les mobilisant, et par là, le regroupement spécifique qu'ils constituent. Il s'agit alors, tout en menant des pratiques conflictuelles et spécifiques, de faire reconnâ̂tre l'identité combative de coopérants en habitation non lucrative, de militants des espaces verts, de femmes du mouvement féministe, de jeunes cherchant la rupture politique avec un ordre social de la domination. On notera finalement que la conquête d'une identité socio-culturelle d'agents sociaux dominés, mobilisés autour d'enjeux aussi nouveaux que spécifiques, n'est vraisemblablement pas sans lien avec l'opposition conflictuelle marquant les tensions contradictoires de l'appropriation étatique et collective du tissu social. De même, elle ne serait point étrangère à la recherche de la véritable libération des pulsions, du moi, de la personnalité.

Un arrêt maintenant s'impose pour faire le point sur cette question des contraintes structurelles, des contradictions des sociétés industrielles avancées avant de conclure en abordant, d'une part, quelques problèmes relatifs à la conscience de l'action et d'autre part, quelques considérations quant à la signification politique de certaines formes de pratique de groupes de base.

Nous avons tout d'abord constaté l'importance des luttes relatives à l'axe distributionnel des ressources au sein de sociétés industrielles avancées faisant face aux problèmes et tensions de 
l'accumulation intensive du capital. Au nom des droits du citoyen, les régimes étatiques et politiques de la démocratie libérale ont été pris d'assaut par des revendications de plus en plus impératives. $\grave{A}$ l'origine de ces tensions entre l'interventionnisme étatique et le fonctionnement de l'État au sein des démocraties libérales, se manifestait l'action sociale non pas de citoyens individualisés, mais de divers groupes d'agents sociaux cherchant la promotion de leurs droits.

Beaucoup de ces groupes, eu égard aux agents plus classiques des oppositions de classes, trahissaient un enracinement, un positionnement social tout à fait autre qui incite à les qualifier de groupes de base ou de mouvements sociaux partiels. Quand leur propension à l'action sociale s'appuie, entre autres, sur leurs conditions socio-économiques de vie, leur positionnement social n'est pas fonction de leur statut de producteurs dans un système de rapports sociaux sous-tendant le procès de production des marchandises. C'est plutôt de leurs rapports à l'État, aux appareils d'État d'où ils tirent le revenu du citoyen, ou encore de leur attribut d'usagers de biens de consommation collective qu'il faut partir si l'on veut comprendre leurs pratiques sociales. Mais leur propension à l'action collective peut aussi être fondée sur leur volonté de conquérir et d'imposer une identité socio-culturelle qui est en correspondance avec les luttes qu'ils mènent à propos d'enjeux conflictuels révélant des rapports de domination socio-politique auxquels ils sont soumis. Ces groupes sociaux contestent alors le mode de production sociale et la domination socio-politique de la jeunesse, de la condition féminine, de la race et de l'ethnie, des orientations sexuelles.

On a souligné précédemment que l'action conflictuelle des mouvements sociaux partiels, des groupes de base, était fréquemment confrontée à cette contradiction où, d'un côté, elle sollicitait la société politique et l'État par l'imposition et la re- 
connaissance de droits collectifs, pavant la voie à un interventionnisme plus diversifié et par là à l'appropriation étatique du tissu social et, de l'autre, elle luttait pour l'affirmation d'un auto-contrôle, d'une autonomie exercée à la base même sur des zones, des aires du tissu social. L'appropriation collective du tissu social visait alors les modes d'habiter, la fréquentation et la consommation d'équipements collectifs, les rapports au corps, l'usage de certains média communaitaires.

L'appropriation collective du tissu social se nourissant à la fois d'une volonté d'auto-contrôle d'aires du tissu social et d'une recherche de solidarité, de sociabilité nouvelles, plus ludiques et communautaires, n'est pas totalement dissociable d'une autre opposition conflictuelle marquant les sociétés industrielles avancées. Des tensions contradictoires apparaissent, en effet, entre la stimulation et la promotion de l'individualité puis, en même temps, les abus marchands de l'individualité et le développement irréductible de l'intégration et du contrôle social. De bien des manières, l'appropriation collective du tissu social pénètre le terrain de l'articulation problématique du culturel et du politique et promeut entre ces régions du social de nouvelles formes de jonction. Elle rejoint alors des enjeux sociaux puis une mobilisation d'agents sociaux qui convergent vers ce mouvement de fond par lequel le privé, la vie privée, souvent devient profondément politique pour les groupes de base des mouvements sociaux partiels concernés ${ }^{15}$.

Bref, autour des contraintes structurelles déjà identifiées et tout juste résumées ci-dessus émergent des enjeux conflictuels majeurs des sociétés industrielles avancées. Force est d'admettre que pour bon nombre de groupes de base et de mouvements

15. Cette tendance à la politisation de la vie privée est notamment présente, comme le souligne à juste titre $\mathrm{S}$. Doré, au sein de branches du mouvement féministe et plus largement encore au sein de certains mouvements contre-culturels; voir S. Doré, Les thérapies féministes, Montréal, Département de sociologie, Université de Montréal, 1983. 
sociaux partiels ces enjeux sont déterminants dans la mesure même où ils révèlent le non-négociable, l'espace ultime de résistance dans un positionnement social dont les caractéristiques de domination sociale face à l'arbitraire économique, mais aussi bien, sinon surtout, politique et culturel, sont de plus en plus nettement identifiées et combattues. On doit d'ailleurs encore souligner que ce fondement en termes de domination sociopolitique aux pratiques de conquête d'une identité socioculturelle, et le rappel qui en est souvent fait dans certaines conjonctures de luttes particulières, provoquent régulièrement le fractionnement de ces groupes de base. Dès lors, ce ne sont plus tous les jeunes, toutes les fermmes, tous les membres d'un même groupe ethnique ou racial qui se mobilisent, mais surtout ceux qui sont plus impliqués dans les rapports de domination sociopolitique et qui manifestent une solidarité et une conscience combatives.

Si donc on cherche à identifier des pratiques de changement social, on retiendra que les conflits auxquels sont mêlés les groupes de base, les mouvements sociaux partiels, ne sauraient aucunement être de ce point de vue sous-estimés. Et pour en tirer des enseignements théoriques et analytiques significatifs, il faut revoir plusieurs des postulats et des outils analytiques appliqués, par exemple, à l'étude des classes et des rapports de classes, aux théories du changement social centrées sur l'État, aux faits de conscience sociale, de conscience de classes, dans les pratiques conflictuelles.

Luttes sociales de groupes de base: problèmes de conscience sociale et nature politique de ces pratiques

Il s'imposait d'accorder une attention soutenue aux problèmes analytiques soulevés par les contraintes structurelles, les 
contradictions des sociétés industrielles avancées qui peuvent mettre en scène, non pas exclusivement mais bien nettement cependant, des groupes de base, des mouvements sociaux partiels et les divers enjeux conflictuels nourissant leurs pratiques sociales. Il ne faudrait point en déduire que toute pratique sociale est directement fonction de contradictions comme si celles-ci, du fait même de leur acuité, menaient tout droit à l'action sociale conflictuelle. Pour l'avoir trop souvent oublié, beaucoup d'analystes de la lutte des classes ont versé dans un déterminisme structurel et un réductionnisme sociologique que le réel social s'est chargé tout naturellement d'invalider. Parmi les facteurs devant mener à l'action, à la mobilisation sociale, les faits de conscience sociale, de conscience de l'action ne sauraient être négligés. Il est hors de notre propos, dans cette conclusion à tirer de quelques développements qui précèdent, de traiter bien longuement de ces questions de faits de conscience sociale, bien que certains aspects de ce thème peuvent être abordés maintenant que nous avons derrière nous certains acquis.

La théorie marxiste contemporaine des rapports de classe a conclu bien nettement, à propos de cette question de la conscience de classe, à la nécessité de dépasser cette fausse dichotomie de la classe en soi et de la classe pour soi; surtout lorsque cette dichotomie reposait sur une approche des rapports de classes qui réservait à la seule condition socio-économique des classes la notion de classe en soi, cette dernière marquant de surcrô̂t la synchronie des rapports de classe, alors que la classe pour soi faisait appel à diverses occurences conjoncturelles de pratiques politiques de classes, pratiques politiques souvent tournées vers la dimension diachronique des rapports de classe ${ }^{16}$. Les efforts analytiques qui ont été faits pour loger dans l'ensemble des contraintes structurelles d'une formation sociale les racines mê-

16. Voir à ce sujet, N. Poulantzas, Pouvoir politique et classes sociales, Paris, Maspéro, 1968, surtout chap. 1. 
mes des rapports de classe ont donc été à l'origine même des effets bénéfiques obtenus par le dépassement de la dichotomie de la classe en soi et de la classe pour soi. Ces deux volets enclenchant une conscience de classe tour à tour fermée sur l'espace plutôt restreint des conditions socio-économiques de vie puis ouverte sur les enjeux du politique, de la transformation sociétale. Et la classe pour soi ne pouvait accéder aux jeux subtils du politique, où elle pouvait d'ailleurs se perdre, sans avoir fondé son assurance dans une conscience bien affermie, bien trempée des rapports d'exploitation économique dont elle était l'objet.

Et quand sont utilisées comme champ de référence d'une analyse des rapports de classe les pratiques socio-conflictuelles des groupes de base, des mouvements sociaux partiels, il s'impose encore davantage, si besoin était, de prendre de la distance par rapport à semblable dichotomie. Que l'identification et la conceptualisation des conditions socio-économiques de vie des agents sociaux de ces groupes de base soient un problème, n'est pas ici le facteur déterminant.

Il faut plutôt mettre en relief cette caractéristique des luttes sociales de certains groupes de base qui veut qu'en plein centre de leurs pratiques sociales conflictuelles ils placent comme enjeu, la conquête d'une identité socio-culturelle de militants luttant pour le contrôle du mode de production sociale de leur identité, de leur être social. Dès lors, dans ce contexte, le privé, la vie privée d'agents sociaux appartenant à ces groupes de base revêt une dimension profondément politique.

Les faits de conscience sociale des agents mobilisés par les enjeux conflictuels de ces groupes de base mènent donc à scruter le champ de la vie quotidienne. Pour beaucoup de ces groupes, la pratique du changement social ne peut être dissociée de l'obligation de changer la vie au plus près du quotidien. C'est sur cette scène particulière que prend racines une pratique de changement social sensible à la conquête d'une identité socio- 
culturelle conditionnée par la nature politique du privé. Et c'est sur cette scène encore que devient tout d'abord manifeste la recherche d'une solidarité affective du groupe, laquelle contribue directement à une sociabilité nouvelle que poursuivent certains groupes de base qui luttent précisément pour la conquête d'une identité socio-culturelle. Nul doute que l'ensemble de ces traits pourrait participer de «l'itinéraire vers le dedans" que $\mathrm{R}$. Bahro considère comme un élément déterminant des ruptures politiques, culturelles, mais aussi de l'émancipation des catégories marchandes, que devraient comporter les véritables perspectives de libération des formes socio-économiques et sociopolitiques de la domination et de l'exploitation.

Un thème doit encore retenir notre attention puisqu'il mène à qualifier la nature politique des pratiques conflictuelles des groupes de base, des mouvements sociaux partiels. Pour certains, serait tout particulièrement frappante cette tendance au refus de l'État. Au delà de la propension de ces pratiques conflictuelles à s'en remettre à l'action directe, par définition très peu médiatisée ce qui, fait problème ici c'est le fait que ces luttes sociales ne développent pas une pratique conflictuelle centrée sur le pouvoir d'État et sa conquête. Pour sûr, pareil décentrage quant au pouvoir d'État n'est pas sans effets troublants par rapport aux pratiques plus traditionnelles de luttes politiques du mouvement ouvrier et par rapport à de multiples théories du changement social basées sur une conceptualisation des rapports de classes et de leurs luttes socio-politiques autour de l'enjeu ultime de la conquête du pouvoir d'État.

S'il est fondé, cependant, de prétendre que les pratiques conflictuelles des mouvements sociaux partiels trouvent racines notamment dans l'opposition contradictoire entre l'appropriation étatique et collective du tissu social et dans la volonté de conquête d'une identité socio-culturelle imposant au régime de la démocratie libérale la reconnaissance de droits collectifs, on 
peut jeter plus d'éclairage sur la nature politique de telles actions sociales. On doit alors mettre tout particulièrement en relief leur très nette propension à provoquer l'éclatement, le débordement du fonctionnement même du système politique. Pourquoi ne pas reconnaitre alors que le débordement du système politique contribue à la remise en cause de la légitimité du pouvoir d'État détenu par les classes politiquement dominantes?

Y a-t-il cependant refus de l'État dans la recherche d'une appropriation collective du tissu social? L'État, est alors sollicité en tant quagence en mesure de contribuer aux revenus du citoyen et apte à fournir divers appuis, même non financiers, les uns et les autres n'en devant pas moins favoriser un autocontrôle, à la base, de zones du tissu social. Auto-contrôle situé au plus loin, et même à l'opposé conflictuel, de l'appropriation étatique du tissu social. Le refus de cet État, de la gestion autoritaire du social, de l'appropriation étatique, suppose-t-il le refus de tout État ou engage-t-il à une redéfinition, dans la pratique conflictuelle même, des rapports entre la société et l'État?

La conjoncture spécifique au système politique québécois est à cet égard bien particulière. Si la quasi-totalité des systèmes politiques contemporains, en ce qui a trait notamment au fonctionnement de la scène politique elle-même, se sont régulièrement montrés imperméables aux demandes et revendications des groupes de base et des mouvements sociaux partiels - ces dernières s'avérant très peu «représentables» sur la scène politique et peu intégrables par les partis politiques ${ }^{17}$ - la situation est

17. Sur ce thème, voir les interrogations sur les rapports entre la société, l'État et le système politique, divergentes bien sûr quant à leurs développements mais convergentes quant à leur thème premier, de $\mathrm{P}$. Rosanvallon, La crise de l'État-providence, Paris, Seuil, 1981, A. Touraine, «La crise de la représentation politique», à paraître, Sociologie et Sociétés, Vol. 15, no. 1, 1983. M. Rioux, "Remarques sur les pratiques émancipatoires dans les sociétés industrielles en crise» dans J.P. Dupuis et al. Les pratiques émancipatoires en milieu populaire Québec, Institut québécois de recherche sur la culture, 1982. 
encore plus extrême au sein de la structure sociale québécoise. Et au moins trois facteurs $\mathrm{y}$ contribuent très directement. Du point de vue même des demandes et des revendications des groupes de base et des mouvements sociaux partiels, le système politique est tout d'abord désarticulé. Des enjeux conflictuels de ces pratiques sociales pénètrent le système politique provincial québécois, d'autres s'inscrivent à la fois dans ce système et dans le système politique fédéral, certains enfin ont tendance à se loger essentiellement au sein de ce dernier système politique. Cette désarticulation du fonctionnement du système politique, tant au niveau de la scène politique que de celui de la société politique et de ses appareils, rend plus grande la distance entre ces pratiques et les instances plus traditionnelles du système politique.

On pourrait alors soutenir, et il n'y a là aucun paradoxe, que les demandes, les revendications des groupes de base, des mouvements sociaux partiels sont rapidement sujets, quand on les examine du point de vue du fonctionnement du système politique, à une sur-politisation. La sur-politisation doit être entendue ici au sens d'une très forte concentration de leur traitement au sein même des appareils exécutifs et administratifs du pouvoir politique provincial et fédéral. Ces pratiques sociales sont directement objet d'un traitement gouvernemental au niveau de ces appareils exécutifs et administratifs, traitement qui n'est point à l'abri des tensions entre appareils et systèmes politiques relatives aux problèmes constitutionnels et aux droits et pouvoir de dépenser, ou de refuser de dépenser, des gouvernements fédéral et provincial. Ce trait de sur-politisation des demandes et des revendications des groupes de base, comporte un corollaire: soit la très nette sous-représentation au niveau des partis politiques, du travail législatif, de la vie politique articulée au suffrage universel - bref au niveau de l'ensemble des composantes de la scène politique au sens strict du terme - des 
revendications et des luttes sociales de ces groupes de base. Il y a là une très grande étanchéité qui systématiquement banalise la vie politique et ne saurait être oubliée quand des formes d'opposition politique prennent des voies parallèles à la scène politique traditionnelle.

Enfin, un tout dernier facteur permet de spécifier la place des revendications et des luttes sociales des groupes de base par rapport au système politique québécois: soit l'absence sur la scène politique, d'un parti politique de gauche. Déjà du point de vue du mouvement ouvrier syndical québécois, pareille absence a eu de grandes conséquences sur les pratiques de lutte de la classe ouvrière elle-même. Et si on considère maintenant les pratiques sociales de ces groupes de base, ce vide politique aura aussi des effets politiques pertinents. Il n'est point étranger, d'une part, au prolongement de ces pratiques, à divers moments de la conjoncture, du côté du mouvement syndical, mais aussi à leurs difficultés notoires d'articulation à ce mouvement, quand il est question de lier les droits des producteurs et des usagers, ou encore les revendications de la classe ouvrière, des travailleurs intellectuels et des groupes de base. On peut noter à cet égard le rôle politique de suppléance, si limité soit-il, que certains tentent de faire jouer au mouvement syndical par rapport aux revendications et aux luttes sociales des groupes de base.

Mais d'autre part, l'absence d'un parti politique de gauche explique cette stratégie des groupuscules politiques qui ont fréquemment tenté de noyauter les milieux militants des groupes de base. Cette tentative aura été fluctuante, mais la conjoncture la plus actuelle donne à penser que la plupart des groupes de base les mieux implantés ont résisté à ce qui a trop souvent pris la forme de tutelles politiques rigides insensibles aux enjeux de leurs luttes. Plus souvent qu'autrement, les orientations théoriques proposées aux pratiques politiques forçaient la concentration de ces luttes, en dernière instance, sur la conquête du pou- 
voir d'État comme théorie et pratique du changement social. Et puis il y eut cette phase de crise aiguë d'une forme de mobilisation par le moyen des groupuscules politiques. Qui pourrait alors nier que les difficultés extrêmes de ces gropuscules à s'adapter à la nature politique spécifique des pratiques conflictuelles des groupes de base et à saisir comment leurs revendications et leurs luttes sociales pouvaient être rendues politiquement représentables, aient directement contribué, parmi d'autres facteurs bien sûr, à leur mise en crise? Curieux effet d'un boomerang politique...

Est-il besoin de souligner, au sujet précisément des problèmes aigus de fonctionnement du système politique, de la scène polirique et de la représentation politique que mettent toujours en relief les revendications, les luttes sociales des groupes de base, combien le défi que ces pratiques socioconflictuelles adressent à la gauche politique québécoise demeure entier. Peut-être est-il plus que temps de sortir des sentiers battus et de chercher sa voie à hauteur d'intelligence et d'audace politique.

Est-il nécessaire d'ajouter que nous ne saurions prétendre que les pratiques conflictuelles des groupes de base, des mouvements sociaux partiels, doivent être conceptualisées comme si ces actions conflictuelles étaient en ce moment même des mieux affirmées et des plus distinctes. Au contraire, nous avons souligné antérieurement que ces pratiques sociales conflictuelles, sont en voie d'émergence et contribuent de plus en plus à la structuration du tissu social de nos sociétés industrielles avancées. Plus ces pratiques conflictuelles s'imposeront, plus il faudra aussi identifier les interrogations et les remises en cause qu'elles provoquent par rapport à la théorie des rapports de classe et du changement social. Et sur ce terrain analytique particulier, il nous faut reconnaitre que ces pratiques conflictuelles amènent à revoir la conceptualisation même des classes sociales, de leurs 
rapports conflictuels et de la conscience de classe. Ces pratiques conflictuelles nous incirent encore à retracer l'impact déterminant des contraintes relatives à la domination socio-politique et à la domination socio-culturelle dans la manière d'analyser et de conceptualiser les rapports conflictuels de classe. Et dans ce contexte, les enjeux conflictuels nourrissant la mobilisation des groupes de base et des mouvements sociaux partiels dirigent notre regard vers les rapports qu'entretiennent, d'un côté, l'État et le système politique, et de l'autre, la société civile. Bref, il faut constater que beaucoup d'aspects des conceptualisations théoriques relatives aux classes et aux rapports de classes doivent être raffinés et revus à la lumière notamment des enseignements qui peuvent être tirés des pratiques conflictuelles en émergence des groupes de base et des mouvements sociaux partiels. Faut-il vraiment s'étonner que la théorie marxiste des classes sociales, qu'il vaudrait beaucoup mieux qualifier de méthodologie, soit à refaire et à raffiner? Au nom même d'une approche dialectique du réel social, ne faut-il pas travailler à la mise à mort, à un moment donné des pratiques théoriques, de ce qui pourrait être qualifié de théorie marxiste des classes sociales? La négation d'un ensemble de propositions théoriques et analytiques qu'il faut constamment reconstruire et raffiner demeure l'étape première d'un travail théorique que la complexité de l'histoire contemporaine impose. 